\title{
Bulk endocytosis at neuronal synapses
}

\author{
NGUYEN Tam $\mathrm{H}^{1}$, QIU XuFeng ${ }^{2}$, SUN JianYuan ${ }^{2} \&$ MEUNIER Frederic $\mathrm{A}^{1^{*}}$ \\ ${ }^{1}$ Queensland Brain Institute, The University of Queensland, Brisbane, Queensland 4072, Australia; \\ ${ }^{2}$ Institute of Biophysics, Chinese Academy of Sciences, Beijing 100101, China \\ Received January 17, 2014; accepted February 24, 2014; published online March 14, 2014
}

\begin{abstract}
Neurotransmitter-containing synaptic vesicle (SV) fusion with the nerve terminal plasma membrane initiates neurotransmission in response to neuronal excitation. Under mild stimulation, the fused vesicular membrane is retrieved via kiss-and-run and/or clathrin-mediated endocytosis, which is sufficient to maintain recycling of SVs. When neurons are challenged with very high stimulation, the number of fused SVs can be extremely high, resulting in significant plasma membrane addition. Under such conditions, a higher capacity retrieval pathway, bulk endocytosis, is activated to redress this large membrane imbalance. Despite first being described more than 40 years ago, the molecular mechanisms underpinning this important process have yet to be clearly defined. In this review, we highlight the current evidence for bulk endocytosis and its prevalence in various neuronal models, as well as discuss the underlying molecular components.
\end{abstract}

bulk endocytosis, synaptic vesicle recycling, synapse, neuron, neurotransmission, stimulation

Citation: $\quad$ Nguyen TH, Qiu XF, Sun JY, Meunier FA. Bulk endocytosis at neuronal synapses. Sci China Life Sci, 2014, 57: 378-383, doi: $10.1007 / \mathrm{s} 11427-014-4636-\mathrm{Z}$

Neurons transmit information by the release of neurotransmitters from a limited pool of synaptic vesicles (SVs) during stimulation-induced exocytosis. During periods of intense stimulation, the rate of vesicle release can reach more than $100 \mathrm{~Hz}$, leading to depletion of SVs. It is therefore essential that nerve terminals have retrieval mechanism(s) to maintain the supply of SVs and sustain neurotransmission. However, which mechanisms are active at synapses to mediate SV membrane retrieval is currently unclear. Studies in various synapse models have demonstrated the involvement of three basic modes of endocytosis: clathrin-mediated endocytosis (CME) [1], kiss-and-run [2,3] and activity- dependent bulk endocytosis (ABDE) [4-8].

CME is the best characterized of the three SV retrieval pathways, due to extensive parallel molecular studies in non-neuronal cells. This pathway retrieves single SVs de novo from the plasma membrane using a set of molecules

*Corresponding author (email: f.meunier@uq.edu.au) including the scaffolding protein clathrin, together with adaptor and accessory proteins [9-11]. The kiss-and-run mode of endocytosis stipulates that SVs do not fully collapse into the plasma membrane and are directly retrieved after the release of neurotransmitter. This pathway involving a transient fusion pore is seemingly compatible with the fast dynamics of a synapse and would also afford energy efficiency due to the transient nature of SV fusion. However, the existence of kiss-and-run in typically small nerve terminals is difficult to assess, with only a few studies published using various fluorescence methods [12-15] or a combination of amperometric and capacitance measurements in neurosecretory cells. One recent study using fluorescent quantal dots to demonstrate differential uptake and release provides promising evidence to support the existence and contribution of kiss-and-run [16]. The third mode of SV retrieval, ADBE, involves the formation of large membrane invaginations or cisternae that undergo fission from the plasma membrane to form internal 'bulk' endo- 
somes. SVs can then bud from these bulk endosomes to replenish the vesicle pools. Under conditions of intense activity, both CME and kiss-and-run are active at central synapses, however, their limited membrane retrieval capacities mean that they are unable to deal with the faster vesicle depletion and large increase in nerve terminal membrane area. ADBE has the capacity to fulfil this requirement for high-capacity membrane retrieval. In this review, we discuss the current evidence for ADBE at different types of neuronal synapses, the techniques used for its detection, and highlight some of the molecular mechanisms driving its function.

\section{ADBE at neuronal synapses}

The first description of ADBE was in a study by Heuser and Reese at the amphibian neuromuscular junction in which they observed the appearance of numerous large intraterminal membrane compartments that they termed 'cisternae' [1], in response to $10 \mathrm{~Hz}$ electrical stimulation for $10 \mathrm{~min}$. As a result of stimulation, the nerve terminals in these preparations contained significantly less synaptic vesicles compared to resting preparations and were morphologically dominated by the presence of the large membrane cisternae. Interestingly, when preparations were rested after $15 \mathrm{~min}$ stimulation, the cisternae disappeared and SVs reappeared, suggesting the SVs could have derived from the cisternae [1]. Since this initial seminal study, a considerable number of studies have demonstrated that ADBE also occurs in rat hippocampal [6,17] and cerebellar granule [18] neurons, lamprey reticulospinal synapses [5], mouse calyx of Held [19] and auditory inner hair cells [20], snake [21] and Caenorhabditis elegans motor terminals [22], and goldfish bipolar neurons [8].

As the name suggests, ADBE occurs in response to elevated synaptic activity $[8,18,23]$. A number of studies have used either pharmacological stimuli at the neuromuscular junction [24] or potassium depolarization in central synapses [25-28] to elicit bulk endocytosis. The use of these non-physiological stimulation methods initially casted doubts on whether bulk endocytosis could be induced under physiological conditions. Subsequent studies, however, showed that bulk endocytosis could be induced in various neuronal preparations including amphibian neuromuscular junction [4], retinal bipolar neurons [8], the calyx of Held [19] and primary cultured neurons [18] using electrical stimulation paradigms within the physiological range encountered in vivo (Figure 1). These demonstrations of ADBE activation by stimulation paradigms mimicking physiological conditions suggest that ADBE is an important SV retrieval pathway during periods of intense synaptic activity. Interestingly, other studies have also shown that ADBE can be triggered using prolonged tetanic simulation [23,31].

\section{Detection of ADBE by membrane capaci- tance measurement}

ADBE has been classically observed using electron microscopic analysis of photoconverted styryl dyes, revealing preferential staining of cisternae by FM1-43 in comparison to FM2-10. This suggests that these structures trap FM1-43 and that the vesicles that slowly bud from them selectively fill the reserve vesicle pool $[4,5,32]$. Likewise, optical imaging of FM dye in retinal bipolar nerve terminals has demonstrated that membrane can be endocytosed into SVs or large cisternae structures [29]. While optical techniques can provide exquisitely high spatial resolution, particularly in the case of electron microscopy, little kinetic information can be obtained. Membrane capacitance measurement, as an assay of membrane surface area, has proved to be a powerful method to monitor SV fusion and retrieval with a high signal and temporal (millisecond time scale) resolution [33]. This approach can further estimate the fusion/fission pore kinetics by monitoring the pore conductance [34]. Capacitance measurements were initially used to monitor vesicle exocytosis and endocytosis in secretory cells and have since been applied to nerve terminals with good success $[35,36]$. Recently, $\mathrm{Wu}$ and $\mathrm{Wu}$ were the first to demonstrate the instant of bulk membrane scission from the plasma membrane in a central synaptic nerve terminal using membrane capacitance measurements [19]. Using calyx of Held synapses in the central nervous system, brief downward capacitance shifts in the range of $20-500 \mathrm{fF}$ were observed from the capacitance traces after strong stimulation (Figure 1D). This correlates to a membrane retrieval that is approximately 2000-fold larger than the capacitance of a single vesicle and consistent with a magnitude that one would expect for ADBE. Moreover, the study found that ADBE occurs within $10 \mathrm{~s}$ after stimulation, much faster than the time scale of minutes as estimated by electron microscopy [4] and optical imaging [29]. These findings demonstrate the existence of ADBE events that are not detectable by electron microscopy or optical imaging due to their low temporal resolution. Importantly, these findings provide evidence that ADBE is a fast mechanism for the clearance of vesicular components from release sites and maintenance of synaptic transmission during sustained strong stimulation [19].

\section{The molecular machinery of ADBE}

Although ADBE was first described more than 40 years ago, little is known about the molecular components and mechanisms underpinning this process. Thus far, only a handful of molecules have been identified as playing roles in various steps of this membrane retrieval pathway (Figure 2). As ADBE is activated in response to intense synaptic activity or stimulation, the molecular sensor(s) that responds to this increased activity is expected to be a critical component, 

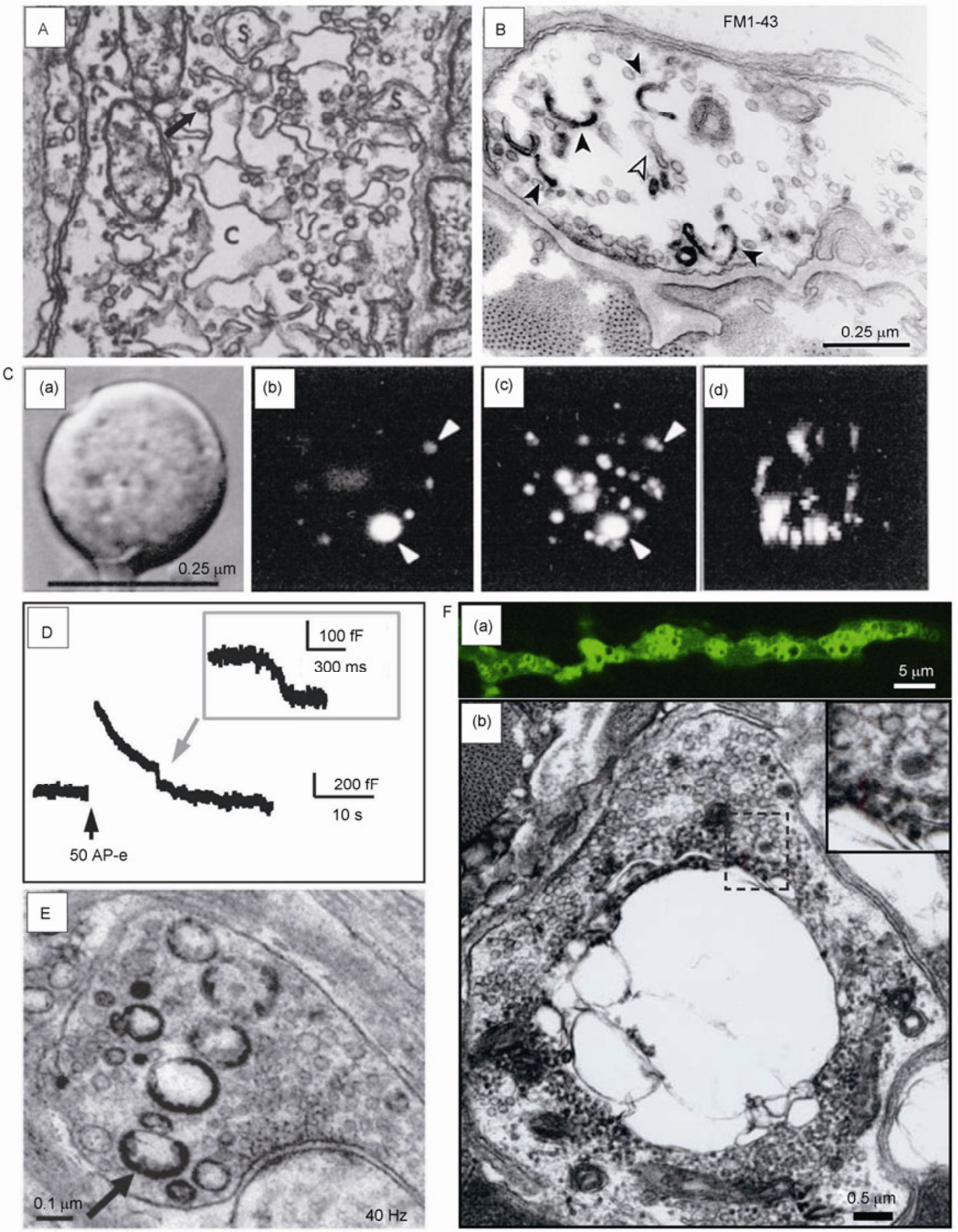

Figure 1 Bulk endocytosis is activated in response to elevated neuronal activity in a variety of neuronal synapses. A, The first description of ADBE in the frog neuromuscular junction elicited by $10 \mathrm{~Hz}$ stimulation for $15 \mathrm{~min}$. Note the numerous large membrane cisternae (C) and coated vesicles (arrow). Schwan cell processes are also present (s). Magnification, 30000× [1]. B, ADBE in frog neuromuscular junction probed using photoconversion of the styryl dye FM1-43 and electron microscopy. $30 \mathrm{~Hz}$ stimulation for $1 \mathrm{~min}$ resulted in the appearance of FM dye in c-shaped bulk cisternae [4]. C(a-d), Bulk endosomes in goldfish retinal bipolar neurons labelled with high molecular weight fluorescent dextran. $\mathrm{C}(\mathrm{b})$ is a single confocal section through the middle of the terminal, $C(c)$ is a projection of a $z$-stack through the entire terminal and $C(d)$ is a projection through the same terminal from the side. Figure reproduced from Holt et al. [29] with permission of The Society for Neuroscience. D, Detection of ADBE using membrane capacitance measurements in mouse Calyx of Held synapses. Fission of bulk endosomes is represented as a large downward capacitance shift (grey arrow and inset) that occurred during compensatory membrane retrieval in response to stimulus of 500 action potential equivalent (AP-e) at $100 \mathrm{~Hz}$ [19]. E, Electron micrographs showing bulk endosomes labelled with HRP (black arrow) in response to 400 action potentials (at $40 \mathrm{~Hz}$ ) stimulation in nerve terminals of cultured rat cerebellar granule neurons. Figure reproduced from Clayton et al. [18] with permission of The Society for Neuroscience. F, Bulk endosomes revealed as large donut-shaped structures by fluorescence imaging of FM1-43 dye (a) and ultrastructural analysis following photoconversion of FM1-43 (b). Note the large number of vesicles containing photoconverted material in close proximity to the delimiting membrane of the bulk cisternae (inset) [30]. 


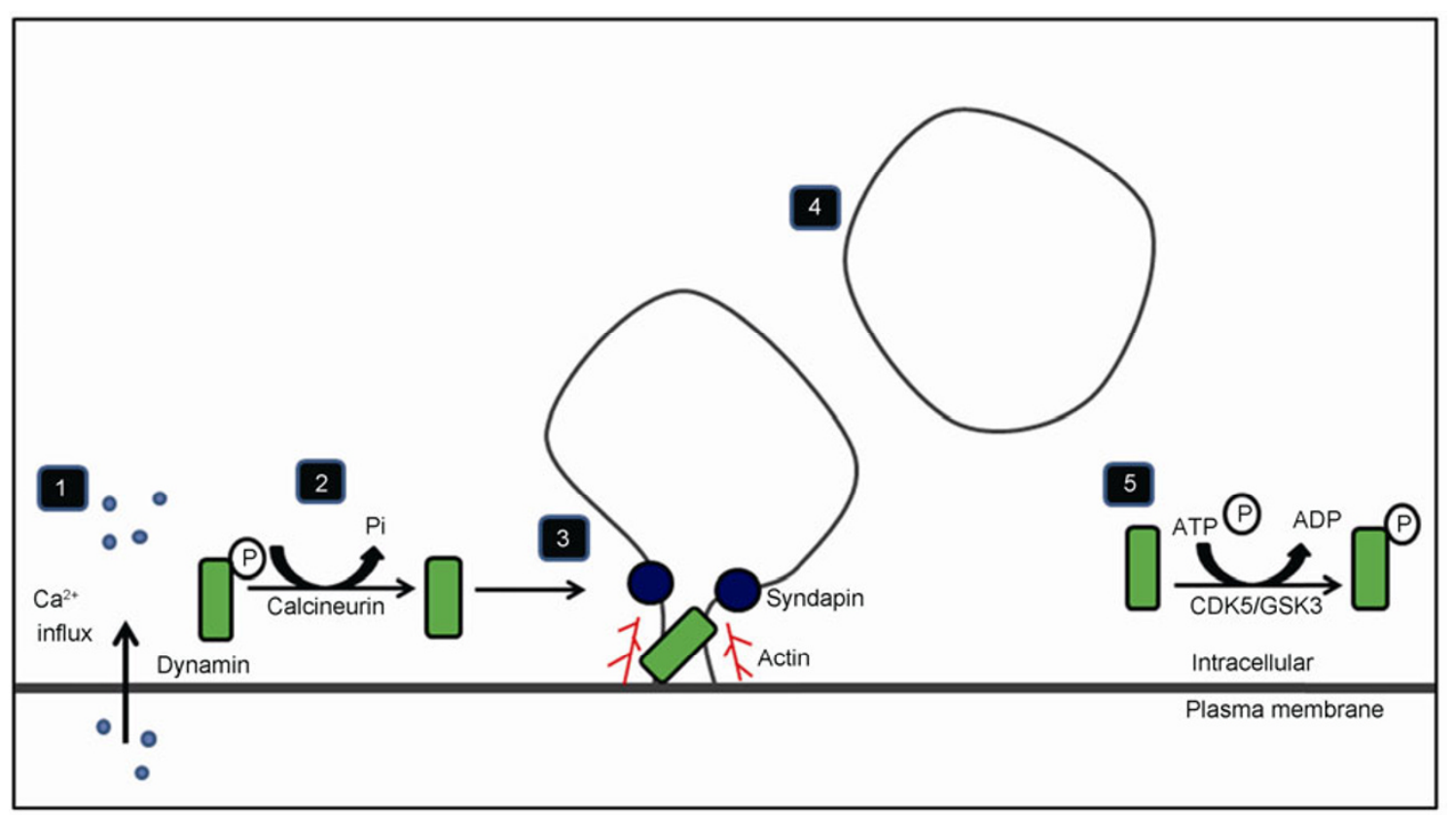

Figure 2 Schematic representation of the interactions between key molecules driving various stages of ADBE. (1) High intensity stimulation results in Ca ${ }^{2+}$ influx into the nerve terminal and activating ADBE sensor molecule, calcineurin. (2) Activated calcineurin dephosphorylates dynamin which then binds syndapin (3) to induce membrane curvature and tabulation to form bulk membrane invagination, a step that may also involve actin. (4) Dynamin mediates the scission of nascent bulk endosomes from the plasma membrane. (5) After scission of bulk endosome, dynamin is rephosphorylated by cyclin dependent kinase 5 (CDK5) and glycogen synthase kinase 3 (GSK3).

with calcineurin emerging as a candidate to fulfil this role. Calcineurin is a calcium-dependent protein phosphatase that dephosphorylates at least eight nerve terminally located substrates, termed the desphophins, all of which are essential for SV endocytosis [37,38]. Two important factors support calcineurin being the sensor molecule for ADBE. Firstly, calcineurin is localized to the cytoplasm and has a low micromolar affinity for calcium [39], meaning that it is not sensitive to the level of calcium influx associated with mild activity and would only encounter sufficient levels of calcium during intense activity. Second, it has been demonstrated that dephosphorylation of one of its key substrates, the large GTPase dynamin I, only occurs at the same stimulation threshold that activates ADBE [40]. Thus, the dephosphorylation function of calcineurin is linked to the activity dependency of ADBE. Indeed, pharmacological disruption of calcineurin function results in inhibition of bulk endocytosis in cultured neurons [28].

Dynamin is a large GTPase protein responsible for the fission of endocytic vesicles from the plasma membrane [41]. Although the role of dynamin in CME is well established, its involvement in bulk endocytosis is less well understood. Evidence implicating dynamin in bulk endocytosis was first obtained in a study by Keonig and Ikeda in nerve terminals of the temperature-sensitive Drosophila shibire mutant $[42,43]$. Shibire flies harbour a temperature-sensitive mutation in the dynamin protein that inactivates the protein at the restrictive temperature $\left(>29^{\circ} \mathrm{C}\right)$ [43]. Keonig and Ikeda's study demonstrated that vesicle recy- cling after synaptic depletion at the restrictive temperature occurs via two pathways, a fast active zone-associated pathway and a slower non-active zone-associated pathway that involves endosomal intermediates. Importantly, tubular invaginations remained connected to the plasma membrane in shibire mutants at the restrictive temperature, indicating that dynamin is required for scission of the large endosomal compartments. While dynamin GTPase activity is required for all forms of SV endocytosis, the dephosphorylation of dynamin is of particular importance in ADBE. As mentioned above, dynamin I is only dephosphorylated by calcineurin during neuronal activity that triggers ADBE [40]. Furthermore, inhibition of the protein kinases responsible for the rephosphorylation of dynamin I after stimulation, cyclin-dependent kinase 5 [28] and glycogen synthase kinase 3 [44], specifically blocks ADBE but not CME. Another important consequence of dynamin dephosphorylation is its interaction with the synaptic protein, syndapin I $[45,46]$. Syndapin, a F-BAR domain-containing protein that is capable of sensing membrane curvature and inducing tabulation [47], may play an active role in driving membrane curvature upon initiation of ADBE. The essential nature of dynamin in ADBE is further demonstrated by the fact that application of the potent inhibitors of dynamin, dynasore [48] and Dyngo $4 \mathrm{a}^{\mathrm{TM}}$ [49], results in the complete block of ADBE both in primary cultured neurons [40] and at the neuromuscular junction [30].

Although the actin cytoskeleton has long been implicated in various steps of the endocytic pathway (reviewed in [50]), 
its role in ADBE is not well understood. The actin cytoskeleton is important in the process of macropinocytosis, the uptake of extracellular fluid in non-neuronal cells [51]. In neuronal cells, actin dynamics are essential for ADBE in both the amphibian neuromuscular junction [30,31] and goldfish retinal bipolar neurons [29]. The involvement of syndapin I in ADBE provides another line of evidence implicating actin in this process. The $\mathrm{SH} 3$ domain of syndapin can not only bind dynamin I, but also the neuronal Wiskott-Aldrich syndrome protein (N-WASP) [52], a protein that recruits the arp2/3 complex to nucleate actin polymerization [53]. Thus, it has been suggested that dephosphorylation-dependent dynamin I recruitment of syndapin I could also recruit N-WASP, and subsequently arp $2 / 3$, to promote actin polymerization at sites of ADBE [54].

Recently, Nguyen et al. demonstrated that both dynamin and actin are differentially required for the development and maturation of ADBE at the amphibian neuromuscular junction [30]. This maturation process was characterized by a transient bulging of the plasma membrane prior to formation of bulk endosomes. The degree of bulging increased with stimulation frequency and the plasmalemma surface retrieved following the transient bulging correlated with the surface membrane internalized in bulk cisternae and recycling vesicles. These findings support a tantalising hypothesis that the maturation process may contribute to a mechanism by which nerve terminals couple exocytosis and bulk endocytosis, by sensing how much presynaptic membrane to retrieve in response to sustained stimulation. However, so far only a correlation between the degree of plasma membrane bulging and the frequency of stimulation has been demonstrated and more work is needed to dissect the full mechanisms underpinning this process.

\section{Concluding remarks}

The molecular mechanisms driving ADBE are still not clearly defined. However, a large body of work during the last decade has shed light on some key aspects of this important SV retrieval pathway. The defining characteristics of bulk endocytosis, high capacity for membrane retrieval and activation in response to synaptic activity, establish this endocytic pathway as an important physiological process in neuronal synapses. Firstly, ADBE provides a mechanism by which nerve terminals can sustain neurotransmission under conditions of high stimulation that can overwhelm the recycling capacity of the CME and/or kiss-and-run vesiclerecycling pathways. Second, it is anticipated that ADBE should be an important component of processes that occur in response to elevated neuronal activity, such as long-term potentiation and synaptic plasticity. Furthermore, ADBE could also play a critical role in neuronal pathologies such as epilepsy, which is characterized by bursts of intense neuronal firing. This is of particular interest as a recent study by Smillie and colleagues showed that ADBE can be blocked by BDNF [55], a neurotrophic factor that has been shown to be released during epileptic seizures [56]. Thus potential therapies designed to target $\mathrm{ADBE}$ are expected to dampen the increased brain activity associated with epilepsy. Such possibilities provide exciting challenges for future research into ADBE, and in particular, the molecular mechanisms that drive this process.

1 Heuser JE, Reese TS. Evidence for recycling of synaptic vesicle membrane during transmitter release at the frog neuromuscular junction. J Cell Biol, 1973, 57: 315-344

2 Ceccarelli B, Hurlbut WP, Mauro A. Turnover of transmitter and synaptic vesicles at the frog neuromuscular junction. J Cell Biol, 1973, 57: 499-524

3 Fesce R, Grohovaz F, Valtorta F, Meldolesi J. Neurotransmitter release: fusion or 'kiss-and-run'? Trends Cell Biol, 1994, 4: 1-4

4 Richards DA, Guatimosim C, Betz WJ. Two endocytic recycling routes selectively fill two vesicle pools in frog motor nerve terminals. Neuron, 2000, 27: 551-559

5 Gad H, Low P, Zotova E, Brodin L, Shupliakov O. Dissociation between $\mathrm{Ca}^{2+}$-triggered synaptic vesicle exocytosis and clathrinmediated endocytosis at a central synapse. Neuron, 1998, 21: 607-616

6 Takei K, Mundigl O, Daniell L, De Camilli P. The synaptic vesicle cycle: a single vesicle budding step involving clathrin and dynamin. J Cell Biol, 1996, 133: 1237-1250

7 Clayton EL, Evans GJ, Cousin MA. Activity-dependent control of bulk endocytosis by protein dephosphorylation in central nerve terminals. J Physiol, 2007, 585: 687-691

8 Paillart C, Li J, Matthews G, Sterling P. Endocytosis and vesicle recycling at a ribbon synapse. J Neurosci, 2003, 23: 4092-4099

9 Sorkin A. Cargo recognition during clathrin-mediated endocytosis: a team effort. Curr Opin Cell Biol, 2004, 16: 392-399

10 Schmid EM, McMahon HT. Integrating molecular and network biology to decode endocytosis. Nature, 2007, 448: 883-888

11 Ford MG, Pearse BM, Higgins MK, Vallis Y, Owen DJ, Gibson A, Hopkins CR, Evans PR, McMahon HT. Simultaneous binding of ptdins(4,5)p2 and clathrin by ap180 in the nucleation of clathrin lattices on membranes. Science, 2001, 291: 1051-1055

12 Klingauf J, Kavalali ET, Tsien RW. Kinetics and regulation of fast endocytosis at hippocampal synapses. Nature, 1998, 394: 581-585

13 Aravanis AM, Pyle JL, Tsien RW. Single synaptic vesicles fusing transiently and successively without loss of identity. Nature, 2003, 423: 643-647

14 Gandhi SP, Stevens CF. Three modes of synaptic vesicular recycling revealed by single-vesicle imaging. Nature, 2003, 423: 607-613

15 Harata NC, Choi S, Pyle JL, Aravanis AM, Tsien RW. Frequency-dependent kinetics and prevalence of kiss-and-run and reuse at hippocampal synapses studied with novel quenching methods. Neuron, 2006, 49: 243-256

16 Zhang Q, Li Y, Tsien RW. The dynamic control of kiss-and-run and vesicular reuse probed with single nanoparticles. Science, 2009, 323: 1448-1453

17 Wenzel EM, Morton A, Ebert K, Welzel O, Kornhuber J, Cousin MA, Groemer TW. Key physiological parameters dictate triggering of activity-dependent bulk endocytosis in hippocampal synapses. PLoS ONE, 2012, 7: e38188

18 Clayton EL, Evans GJ, Cousin MA. Bulk synaptic vesicle endocytosis is rapidly triggered during strong stimulation. J Neurosci, 2008, 28: 6627-6632

$19 \mathrm{Wu} \mathrm{W}, \mathrm{Wu}$ LG. Rapid bulk endocytosis and its kinetics of fission pore closure at a central synapse. Proc Natl Acad Sci USA, 2007, 104: 10234-10239

20 Neef J, Jung S, Wong AB, Reuter K, Pangrsic T, Chakrabarti R, 
Kügler S, Lenz C, Nouvian R, Boumil RM, Frankel WN, Wichmann $\mathrm{C}$, Moser T. Modes and regulation of endocytic membrane retrieval in mouse auditory hair cells. J Neurosci, 2014, 34: 705-716

21 Teng H, Lin MY, Wilkinson RS. Macroendocytosis and endosome processing in snake motor boutons. J Physiol, 2007, 582: 243-262

22 Kittelmann M, Liewald JF, Hegermann J, Schultheis C, Brauner M, Steuer Costa W, Wabnig S, Eimer S, Gottschalk A. In vivo synaptic recovery following optogenetic hyperstimulation. Proc Natl Acad Sci USA, 2013, 110: E3007-3016

23 Andersson F, Jakobsson J, Low P, Shupliakov O, Brodin L. Perturbation of syndapin/pacsin impairs synaptic vesicle recycling evoked by intense stimulation. J Neurosci, 2008, 28: 3925-3933

24 Miller TM, Heuser JE. Endocytosis of synaptic vesicle membrane at the frog neuromuscular junction. J Cell Biol, 1984, 98: 685-698

25 Marxen M, Volknandt W, Zimmermann H. Endocytic vacuoles formed following a short pulse of $\mathrm{K}^{+}$-stimulation contain a plethora of presynaptic membrane proteins. Neuroscience, 1999, 94: 985-996

26 Leenders AG, Scholten G, de Lange RP, Lopes da Silva FH, Ghijsen WE. Sequential changes in synaptic vesicle pools and endosome-like organelles during depolarization near the active zone of central nerve terminals. Neuroscience, 2002, 109: 195-206

27 de Lange RP, de Roos AD, Borst JG. Two modes of vesicle recycling in the rat calyx of held. J Neurosci, 2003, 23: 10164-10173

28 Evans GJ, Cousin MA. Activity-dependent control of slow synaptic vesicle endocytosis by cyclin-dependent kinase 5. J Neurosci, 2007, 27: 401-411

29 Holt M, Cooke A, Wu MM, Lagnado L. Bulk membrane retrieval in the synaptic terminal of retinal bipolar cells. J Neurosci, 2003, 23: $1329-1339$

30 Nguyen TH, Maucort G, Sullivan RK, Schenning M, Lavidis NA, McCluskey A, Robinson PJ, Meunier FA. Actin- and dynamin-dependent maturation of bulk endocytosis restores neurotransmission following synaptic depletion. PLoS ONE, 2012, 7: e36913

31 Richards DA, Rizzoli SO, Betz WJ. Effects of wortmannin and latrunculin A on slow endocytosis at the frog neuromuscular junction. J Physiol, 2004, 557: 77-91

32 Henkel AW, Lubke J, Betz WJ. Fm1-43 dye ultrastructural localization in and release from frog motor nerve terminals. Proc Natl Acad Sci USA, 1996, 93: 1918-1923

33 Neher E, Marty A. Discrete changes of cell membrane capacitance observed under conditions of enhanced secretion in bovine adrenal chromaffin cells. Proc Natl Acad Sci USA, 1982, 79: 6712-6716

34 Rosenboom H, Lindau M. Exo-endocytosis and closing of the fission pore during endocytosis in single pituitary nerve terminals internally perfused with high calcium concentrations. Proc Natl Acad Sci USA, 1994, 91: 5267-5271

35 von Gersdorff H, Matthews G. Dynamics of synaptic vesicle fusion and membrane retrieval in synaptic terminals. Nature, 1994, 367: 735-739

36 Sun JY, Wu LG. Fast kinetics of exocytosis revealed by simultaneous measurements of presynaptic capacitance and postsynaptic currents at a central synapse. Neuron, 2001, 30: 171-182

37 Cousin MA, Robinson PJ. The dephosphins: dephosphorylation by calcineurin triggers synaptic vesicle endocytosis. Trends Neurosci, 2001, 24: 659-665

38 Lee SY, Voronov S, Letinic K, Nairn AC, Di Paolo G, De Camilli P. Regulation of the interaction between pipki gamma and talin by pro- line-directed protein kinases. J Cell Biol, 2005, 168: 789-799

39 Klee CB, Crouch TH, Krinks MH. Calcineurin: a calcium- and calmodulin-binding protein of the nervous system. Proc Natl Acad Sci USA, 1979, 76: 6270-6273

40 Clayton EL, Anggono V, Smillie KJ, Chau N, Robinson PJ, Cousin MA. The phospho-dependent dynamin-syndapin interaction triggers activity-dependent bulk endocytosis of synaptic vesicles. J Neurosci, 2009, 29: 7706-7717

41 Marks B, Stowell MH, Vallis Y, Mills IG, Gibson A, Hopkins CR, McMahon HT. Gtpase activity of dynamin and resulting conformation change are essential for endocytosis. Nature, 2001, 410: 231-235

42 Koenig JH, Ikeda K. Synaptic vesicles have two distinct recycling pathways. J Cell Biol, 1996, 135: 797-808

43 van der Bliek AM, Meyerowitz EM. Dynamin-like protein encoded by the Drosophila shibire gene associated with vesicular traffic. Nature, 1991, 351: 411-414

44 Smillie KJ, Cousin MA. Akt/pkb controls the activity-dependent bulk endocytosis of synaptic vesicles. Traffic, 2012, 13: 1004-1011

45 Qualmann B, Roos J, DiGregorio PJ, Kelly RB. Syndapin I, a synaptic dynamin-binding protein that associates with the neural wiskott-aldrich syndrome protein. Mol Biol Cell, 1999, 10: 501-513

46 Anggono V, Smillie KJ, Graham ME, Valova VA, Cousin MA, Robinson PJ. Syndapin I is the phosphorylation-regulated dynamin I partner in synaptic vesicle endocytosis. Nat Neurosci, 2006, 9: $752-760$

47 Itoh T, Erdmann KS, Roux A, Habermann B, Werner H, De Camilli P. Dynamin and the actin cytoskeleton cooperatively regulate plasma membrane invagination by bar and F-bar proteins. Dev Cell, 2005, 9: 791-804

48 Macia E, Ehrlich M, Massol R, Boucrot E, Brunner C, Kirchhausen T. Dynasore, a cell-permeable inhibitor of dynamin. Developmental cell, 2006, 10: 839-850

49 McCluskey A, Daniel JA, Hadzic G, Chau N, Clayton EL, Mariana A, Whiting A, Gorgani NN, Lloyd J, Quan A, Moshkanbaryans L, Krishnan S, Perera S, Chircop M, von Kleist L, McGeachie AB, Howes MT, Parton RG, Campbell M, Sakoff JA, Wang X, Sun JY, Robertson MJ, Deane FM, Nguyen TH, Meunier FA, Cousin MA, Robinson PJ. Building a better dynasore: the dyngo compounds potently inhibit dynamin and endocytosis. Traffic, 2013, 14: 1272-1289

50 Mooren OL, Galletta BJ, Cooper JA. Roles for actin assembly in endocytosis. Ann Rev Biochem, 2012, 81: 661-686

51 Nichols BJ, Lippincott-Schwartz J. Endocytosis without clathrin coats. Trends Cell Biol, 2001, 11: 406-412

52 Kessels MM, Qualmann B. Syndapins integrate N-WASP in receptor-mediated endocytosis. EMBO J, 2002, 21: 6083-6094

53 Mullins RD, Heuser JA, Pollard TD. The interaction of Arp2/3 complex with actin: nucleation, high affinity pointed end capping, and formation of branching networks of filaments. Proc Natl Acad Sci USA, 1998, 95: 6181-6186

54 Clayton EL, Cousin MA. The molecular physiology of activity-dependent bulk endocytosis of synaptic vesicles. J Neurochem, 2009, 111: 901-914

55 Smillie KJ, Pawson J, Perkins EM, Jackson M, Cousin MA. Control of synaptic vesicle endocytosis by an extracellular signalling molecule. Nat Commun, 2013, 4: 2394

56 Koyama R, Ikegaya Y. To BDNF or not to BDNF: that is the epileptic hippocampus. Neuroscientist, 2005, 11: 282-287

Open Access This article is distributed under the terms of the Creative Commons Attribution License which permits any use, distribution, and reproduction in any medium, provided the original author(s) and source are credited. 